\title{
Quantization and Morita Equivalence for Constant Dirac Structures on Tori
}

\author{
Xiang Tang and Alan Weinstein* \\ Department of Mathematics \\ University of California \\ Berkeley, CA 94720 USA \\ (xtang@math.berkeley.edu, alanw@math.berkeley.edu)
}

\begin{abstract}
We define a $C^{*}$-algebraic quantization of constant Dirac structures on tori, which extends the standard quantization of Poisson structures. We prove that Dirac structures in the same orbit of a natural action of $O(n, n \mid \mathbb{Z})$ give rise to Morita equivalent algebras, completing and extending a theorem of Rieffel and Schwarz.
\end{abstract}

\section{Introduction}

Quantum, or noncommutative, tori can be obtained by deformation quantization of constant Poisson structures on ordinary tori. This fact was noticed by the second author 19 and was developed by Rieffel [15] into a rigorous theory of "strict deformation quantization" from Poisson manifolds to $C^{*}$ algebras. Earlier, Rieffel 12 had introduced a notion of strong Morita equivalence for operator algebras, sufficient to imply the equivalence of suitable categories of topological representations. For simplicity, we shall refer to this notion simply as "Morita equivalence."

Motivated by applications to duality in string theory (see [17]), Rieffel and Schwarz [16 showed, with an additional technical hypothesis, that the algebras of functions on two noncommutative $n$-tori are Morita equivalent if the underlying Poisson structures are related by a "fractional linear transformation" whose coefficient matrix belongs to $S O(n, n \mid \mathbb{Z})$. In this paper, we will prove the Rieffel-Schwarz result without the additional hypothesis by extending the scope of the theorem from Poisson structures to Dirac structures, whose definition we will recall later in this introduction.

$\mathrm{Li}[8]$ has also proven the full theorem for the case of Poisson structures, and in fact all of our results could be deduced from his theorem. Nevertheless, our proof is completely different, and we believe that it sheds further light on the role of $S O(n, n \mid \mathbb{Z})$.

A constant Poisson structure on $\mathbb{T}^{n}=\mathbb{R}^{n} / \mathbb{Z}^{n}$ is specified by a skew-symmetric, real, $n \times n$ matrix $\Pi$, representing a skew-symmetric bilinear form on the space $\mathbb{R}^{n *}$ of translation-invariant vector fields on the torus. The standard deformation quantization of the Poisson manifold $\left(\mathbb{T}^{n}, \Pi\right)$ is obtained by deforming the algebra of finite Fourier series on $\mathbb{T}^{n}$ using the multiplication rule

$$
e_{r} *_{\hbar} e_{s}=e^{-\pi i \hbar \Pi(r, s)} e_{r+s}
$$

where $r$ and $s$ are multi-indices in $\mathbb{Z}^{n}, \hbar$ is the deformation parameter, and $e_{m}(x)=e^{2 \pi i m x}$. This product extends to the Fourier series with rapidly decreasing coefficients and from there to a $C^{*}$ completion $\mathcal{A}_{\hbar \Pi}$ which is known as "the algebra of continuous functions on the quantum torus ${ }^{1} \mathbb{T}_{\hbar \Pi}$ ". At this point, we

\footnotetext{
${ }^{*}$ Research of both authors partially supported by NSF Grant DMS-02-04100.

MSC2000 Subject Classification Numbers: 46L65,81S10 (Primary).

Keywords: Dirac structure, Poisson structure, Morita equivalence, quantization.

${ }^{1}$ Sometimes $\mathcal{A}_{\hbar \Pi}$ is itself referred to as the quantum torus, but since this terminology does not agree with normal usage when $\Pi=0$, we prefer not to use it.
} 
will leave the world of deformations by setting $\hbar=1$ so that we have, for each skew-symmetric form $\Pi$, the algebra $\mathcal{A}_{\Pi}$ of functions on $\mathbb{T}_{\Pi}$. This algebra may also be described as the $C^{*}$-algebra determined by $n$ unitary generators $\epsilon_{1}, \ldots, \epsilon_{n}$ (lower indices here are in $\mathbb{Z}$ rather than $\mathbb{Z}^{n}$ ) subject to the commutation relations $\epsilon_{j} \epsilon_{i}=e^{-2 \pi i \hbar \Pi_{i j}} \epsilon_{i} \epsilon_{j}$.

One sees immediately from the commutation relations that two kinds of operations on $\Pi$. do not change the isomorphism class of $\mathcal{A}_{\Pi}$. Adding a matrix with integer coefficients does not change the algebra at all. Also, if we $A \in G L(n, \mathbb{Z})$, then the algebras $\mathcal{A}_{\Pi}$ and $\mathcal{A}_{A \Pi A^{t}}$ are isomorphic via the map which takes $\epsilon_{i}$ to $\prod_{j} \epsilon_{j}^{a_{i j}}$.

Much less obvious are operations for which the quantized algebras are not isomorphic but are still Morita equivalent. The first of these was discovered by Rieffel [13] when $n=2$. The matrix $\Pi$ then has the form $\left(\begin{array}{cc}0 & \theta \\ -\theta & 0\end{array}\right)$, where $\theta$ is a real number. The first of the two kinds of operation above adds an integer to $\theta$, while the second leaves $\theta$ fixed or simply changes its sign. Rieffel proved in [13] that a third operation, namely replacing $\theta$ by $1 / \theta$, preserves the Morita equivalence class of the algebra. The three types of operations are contained in, and in fact generate, the action of the group $G L(2, \mathbb{Z})$ on the real numbers by fractional linear transformations $\theta \mapsto(p \theta+q)(r \theta+s)^{-1}$; hence, the algebras corresponding to $\theta$ and $\theta^{\prime}$ are Morita equivalent if $\theta$ and $\theta^{\prime}$ are in the same $G L(2, \mathbb{Z})$ orbit. The converse is also proved in 13 .

To a large extent, the main result of [16] extends to higher dimensional tori the "if" part of the classification above (but not the converse, which is false unless $n=2$ ). The group $O(n, n \mid \mathbb{R})$ of automorphisms of $\mathbb{R}^{n} \oplus \mathbb{R}^{n *}$ preserving the indefinite inner product

$$
\left(X_{1}+\xi_{1}, X_{2}+\xi_{2}\right)=\frac{1}{2}\left(<\xi_{1}, X_{2}>+<\xi_{2}, X_{1}>\right)
$$

has subgroups $O(n, n \mid \mathbb{Z})$ and $S O(n, n \mid \mathbb{Z})$ defined in the obvious way. An element of $O(n, n \mid \mathbb{Z})$ may be written in block form as

$$
g=\left(\begin{array}{ll}
A & B \\
C & D
\end{array}\right),
$$

where $A, B, C$, and $D$ are $n \times n$ integer matrices which satisfy $A^{t} C+C^{t} A=0=B^{t} D+D^{t} B$ and $A^{t} D+C^{t} B=1$. Such a matrix "acts" on the space $\mathcal{T}_{n}$ of skew-symmetric $n \times n$ matrices by taking $\Pi$ to

$$
g \cdot \Pi=(A \Pi+B)(C \Pi+D)^{-1} .
$$

The word "acts" is in quotation marks because the right hand side is not defined when the denominator $C \Pi+D$ is singular.

The main result of [16] may now be stated as follows.

Theorem 1.1. If $\Pi \in \mathcal{T}_{n}$ is such that $g \cdot \Pi$ is defined for all $g \in S O(n, n \mid \mathbb{Z})$, then the algebras $\mathcal{A}_{g} \Pi$ are all Morita equivalent to one another.

We note that [16] contains a counterexample to the converse of this theorem when $n=3$, while Schwarz 17] proves a converse using a refined notion known as "complete Morita equivalence."

The proof of Theorem 1.1 in [16] uses a decomposition of the general element $g \in S O(n, n \mid \mathbb{Z})$ as a product of generators of three types, analogous to the three types described above for $n=2$. As a result, it does not establish the Morita equivalence of $\Pi$ and $g_{0} \cdot \Pi$ if the action on $\Pi$ is defined for a particular $g_{0}$ but not for all $g$. The key idea of the present paper is to circumvent this difficulty by enlarging (in fact, compactifying $) \mathcal{T}_{n}$ to the space of Dirac structures, on which the action of $O(n, n \mid \mathbb{R})$, and hence that of $S O(n, n \mid \mathbb{Z})$, is everywhere defined. This idea was suggested by the appearance of the bilinear form (2) in both the Rieffel-Schwarz theorem and the definition by Courant 4 of Dirac structures, which we now recall.

Definition 1.2. A Dirac structure on a vector space $V$ is a maximal isotropic subspace of $V \oplus V^{*}$ with respect to the non-degenerate symmetric bilinear form (2). A Dirac structure on a vector bundle E is a subbundle of $E \oplus E^{*}$ which is a Dirac structure on each fibre. A Dirac structure on a manifold $M$ is a Dirac structure on TM whose space of sections is closed under the Courant bracket

$$
\left[X_{1}+\xi_{1}, X_{2}+\xi_{2}\right]=\left[X_{1}, X_{2}\right]+L_{X_{1}} \xi_{2}-L_{X_{2}} \xi_{1}+\frac{1}{2} d\left(<\xi_{1}, X_{2}>-<\xi_{2}, X_{1}>\right)
$$


The space of all Dirac structures on the vector space $\mathbb{R}^{n}$ will be denoted by $\mathcal{D}_{n}$.

Dirac structures on a manifold $M$ include the Poisson structures and closed 2-forms (identified with the graphs of the corresponding skew-symmetric bundle maps $T^{*} M \rightarrow T M$ or $T M \rightarrow T^{*} M$ ), as well as the foliations (identified with direct sums $F \oplus F^{\circ}$, where $F$ is an integrable subbundle of $T M$, and $F^{\circ}$ is its annihilator).

From now on, we will be concerned exclusively with constant (i.e. translation-invariant) Dirac structures on tori $\mathbb{T}^{n}$. Since all the terms in the Courant bracket involve derivatives, these are just the translation-invariant Dirac structures on the tangent bundle $T \mathbb{T}^{n}$, or equivalently the Dirac structures on the vector space $\mathbb{R}^{n}$ of constant vector fields on $\mathbb{T}^{n}$ (which may be identified with the tangent space at any point).

The "action" of $O(n, n \mid \mathbb{R})$ on antisymmetric matrices now has the following interpretation. We identify each $\Pi \in \mathcal{T}_{n}$ with the map $\mathbb{R}^{n *} \rightarrow \mathbb{R}^{n}$ of which it is the matrix with respect to the standard basis and its dual. Then we identify $\Pi$ with its graph

$$
\Gamma_{\Pi}=\left\{(\Pi \xi, \xi) \mid \xi \in \mathbb{R}^{n *}\right\},
$$

an element of $\mathcal{D}_{n}$. The group $O(n, n \mid \mathbb{R})$ acts in the obvious way on $\mathcal{D}_{n}$, and the correspondence $\Pi \mapsto \Gamma_{\Pi}$ is $O(n, n \mid \mathbb{R})$ equivariant with respect to the action (3). Thus, if a product of generators $g=g_{r} \cdots g_{1}$ maps $\Pi$ to $\Pi^{\prime}$, even if $g_{1} \Pi, g_{2} g_{1} \Pi, \ldots$ are not all defined as antisymmetric matrices, they are defined as Dirac structures. Our strategy for proving the Rieffel-Schwarz theorem, then, is to attach an algebra $\mathcal{A}_{\Gamma}$ (more precisely, a Morita equivalence classes of algebras) to each Dirac structure $\Gamma$, and to prove that this Morita equivalence class is unchanged when $\Gamma$ is transformed by any member of a certain set of generators of $O(n, n \mid \mathbb{Z})$.

Remark 1.3. The apparent extension of the Rieffel-Schwarz theorem from $S O(n, n \mid \mathbb{Z})$ to $O(n, n \mid \mathbb{Z})$ is illusory. As we will see in Corollary 2.3 below any $g \in O(n, n \mid \mathbb{Z})$ which transforms some Poisson structure into another one must lie in $S O(n, n \mid \mathbb{Z})$. On the other hand, by passing to $O(n, n \mid \mathbb{Z})$, we will not only bypass the "obstruction" in the original proof, but we will reduce from three to two the number of kinds of generators which must be dealt with.

To construct $\mathcal{A}_{\Gamma}$, we begin by recalling from [4] (Proposition 1.1.4) that to every Dirac structure $\Gamma$ on the vector space $\mathbb{R}^{n}$ there corresponds a natural bivector $\Pi_{\Gamma}$ on the quotient of $\mathbb{R}^{n}$ by $\mathbb{R}^{n} \cap \Gamma$. (Here, we are identifying $\mathbb{R}^{n}$ with the subspace $\mathbb{R}^{n} \oplus\{0\}$ of $\mathbb{R}^{n} \oplus \mathbb{R}^{n *}$.) Conversely (with a proof similar to arguments in Section 1.1 of [4), the intersection $\mathbb{R}^{n} \cap \Gamma$ and the bivector $\Pi_{\Gamma}$ determine $\Gamma$.

For a constant Dirac structure $\Gamma$ on $\mathbb{T}^{n}$, the intersection $\mathbb{R}^{n} \cap \Gamma$ defines a foliation known as the characteristic foliation of the Dirac structure, while the bivector $\Pi_{\Gamma}$ defines a Poisson structure transverse to this foliation. The idea behind what follows is that the function algebra of the quantized Dirac manifold $\left(\mathbb{T}^{n}, \Gamma\right)$ should be obtained from a foliation algebra for the characteristic foliation (specifically, the groupoid algebra of the leafwise fundamental groupoid of the foliation) by "deformation" via the transverse Poisson structure. This idea, already suggested by Block and Getzler [1] (see also [20]), is at best inconvenient to apply directly in our setting, since it requires the extra data of a so-called Haar form. Instead, we restrict the fundamental groupoid of the foliation to a subtorus $M \subseteq \mathbb{T}^{n}$ which is a complete transversal to obtain an equivalent étale groupoid. $M$ carries a Poisson structure $\Pi_{\Gamma, M}$, from which we can obtain a quantum torus algebra $\mathcal{A}_{\Pi_{\Gamma, M}}$ in the usual way. The restricted groupoid turns out to be a transformation groupoid for an action of a lattice on $M$, and so we may form the crossed product algebra of $\mathcal{A}_{\Pi_{\Gamma, M}}$ with the lattice. This algebra depends on the choice of $M$, but we will show that its Morita equivalence class depends only on $\Gamma$. This "independence of transversal" proof, carried out in detail (in a more general context) in [18, is the only analytic ingredient in our proof. Its use is thus an extension of the idea of Connes (Section $8 . \beta$ in [2]), that the original Rieffel theorem for $\mathbb{T}^{2}$ is a consequence of the Morita invariance of ordinary foliation groupoid algebras under change of transversal.

Acknowledgments: We would like to thank H. Bursztyn, M. Crainic, K. Fukaya, H. Li, M. Rieffel and A. Schwarz for helpful suggestions.

\section{Linear algebra of Dirac structures}

We begin with some notation and definitions related to Dirac structures on a vector space $V$. 
We will identify $V$ with the subspace $V \oplus 0$ of $V \oplus V^{*}$. The projection $V \oplus V^{*} \rightarrow V^{*}$ will be denoted by $p_{*}$. The annihilator in $V^{*}$ of a subspace $E \subseteq V$ will be denoted by $V^{\circ}$.

Definition 2.1. The characteristic subspace $C(\Gamma)$ of a Dirac structure $\Gamma$ on $V$ is $\Gamma \cap V$. The dimension of $C(\Gamma)$ is the nullity $N(\Gamma)$ of $\Gamma$. $\Gamma$ is called even or odd according to the dimension of its nullity.

The maximal isotropic property of $\Gamma$ immediately implies that

$$
C(\Gamma)^{\circ}=p_{*}(\Gamma) .
$$

Lemma 2.2. The spaces of even and odd Dirac structures are the connected components of $\mathcal{D}_{n}$. The action of $S O(n, n \mid \mathbb{R})$ leaves each component invariant, while the action of $O(n, n \mid \mathbb{R}) \backslash S O(n, n \mid \mathbb{R})$ interchanges the components.

Proof. We follow Courant [4] and rewrite $\mathbb{R}^{n} \oplus \mathbb{R}^{n *}$ as an orthogonal direct sum $P \oplus N$, by choosing a positive definite symmetric inner product $b$ on $\mathbb{R}^{n}$ and letting $P$ be the graph of the corresponding map $\mathbb{R}^{n} \rightarrow \mathbb{R}^{n *}$. Its orthogonal complement $N$ is the graph of the map corresponding to $-b . P$ and $N$ are maximal positive definite and negative subspaces of $\mathbb{R}^{n} \oplus \mathbb{R}^{n *}$, and the Dirac structures on $\mathbb{R}^{n}$ are the graphs of anti-isometries from $P$ to $N$, with which they may be identified. If we fix one such anti-isometry $K$, namely the one identified with $\mathbb{R}^{n} \oplus\{0\}$, each Dirac structure $\Gamma$ may be identified by composition with $K^{-1}$ with an isometry $L$ from $P$ to itself, and the characteristic subspace of $\Gamma$ is then identified with the fixed space of $L$. Since the codimension of the fixed space of $L$ is even or odd according to whether $L$ preserves or reverses orientation, the dimension modulo 2 of the characteristic subspace is constant on each component of $\mathcal{D}_{n}$.

To analyze the effect of $O(n, n \mid \mathbb{R})$ on parity, it suffices to look at the maximal compact subgroup $O(n) \times O(n)$ which leaves the subspaces $P$ and $N$ invariant. An element of this subgroup is a pair $(A, B)$ of isometries of $P$ and $N$ respectively, and it takes the Dirac structure associated with $K^{-1} L: P \rightarrow P$ to the structure associated with $K^{-1} B L A^{-1}$. The last part of the lemma now follows from the identity $\operatorname{det} K^{-1} B L A^{-1}=\operatorname{det} K^{-1} L \operatorname{det} B \operatorname{det} A$.

Since Poisson structures are even Dirac structures, we have the following corollary, which gives one explanation for the appearance of the special orthogonal group in the Rieffel-Schwarz theorem.

Corollary 2.3. Any element of $O(n, n \mid \mathbb{R})$ which transforms one Poisson structure to another must lie in $S O(n, n \mid \mathbb{R})$.

We now introduce further notation in $\mathbb{R}^{n}$ and $O(n, n \mid \mathbb{Z})$. Let $\left(e_{1}, \ldots, e_{n}\right)$ and $\left(f_{1}, \ldots f_{n}\right)$ be the standard basis of $\mathbb{R}^{n}$ and its dual basis. For any subset $I$ of $\{1,2, \ldots, n\}$, we denote its complement by $I^{\prime}$ and its cardinality by $|I| . \mathbb{R}^{I}$ and $\mathbb{R}^{I^{*}}$ will denote the subspaces of $\mathbb{R}^{n}$ and $\mathbb{R}^{n *}$ spanned by the $e_{i}$ (or $\left.f_{i}\right)$ for $i \in I$. Note that $\left(\mathbb{R}^{I}\right)^{\circ}=\mathbb{R}^{I^{\prime *}}$. We will also use this "exponent" notation for subgroups of $\mathbb{Z}^{n}$ and $\mathbb{T}^{n}$.

The element of $O(n, n \mid \mathbb{Z})$ which exchanges $e_{i}$ with $f_{i}$ for all $i \in I$ and which leaves all other basis elements fixed will be denoted by $\sigma_{I}$. If $A$ belongs to $G L(n, \mathbb{Z})$, we will denote by $\rho(A)$ the element of $O(n, n \mid \mathbb{Z})$ which acts on the first summand of $\mathbb{R}^{n} \oplus \mathbb{R}^{n *}$ by $A$ and on the second summand by $\left(A^{t}\right)^{-1}$. If $N$ is a skew symmetric $n \times n$ matrix with integer entries, $\nu(N)$ will denote the map $(x, y) \mapsto(x+N y, y)$. (When applied as a fractional linear transformation, $\nu(N)$ just adds $N$ to each Poisson structure.) The additive group of all $\nu(N)$ 's is generated by its elements $\nu_{i j}$ for $i<j$, where $\nu_{i j}$ is the sum of the identity with the rank 2 matrix which maps $f_{i}$ to $e_{j}, f_{j}$ to $-e_{i}$, and all other basis elements to zero.

It is proven in [16] that $S O(n, n \mid \mathbb{Z})$ is generated by the $\rho(A)$ 's, the $\nu_{i j}$ 's, and $\sigma_{\{1,2\}}$. It follows easily that $O(n, n \mid \mathbb{Z})$ is generated by the $\rho(A)$ 's, the $\nu_{i j}$ 's, and $\sigma_{\{1\}}$. But in fact even more is true.

Lemma 2.4. The group $O(n, n \mid \mathbb{Z})$ is generated by $\sigma_{\{1\}}$ and $\rho(G L(n, \mathbb{Z}))$.

Proof. The subgroup generated by $\sigma_{\{1\}}$ and $\rho(G L(n, \mathbb{Z}))$ contains $\sigma_{\{i\}}$ for all $i$. Hence it contains $\sigma_{\{1\}} \sigma_{\{2\}}=\sigma_{\{1,2\}}$. A straightforward computation shows that $\nu_{i j}=\sigma_{\{i\}} \rho(A) \sigma_{\{i\}}$, where $A$ maps $e_{i}$ to $e_{i}+e_{j}$ and fixes all the other basis elements of $\mathbb{R}^{n}$.

Remark 2.5. Elimination of the generators $\nu_{i j}$ will be essential in our proof. Although these generators act trivially on the quantization of Poisson structures, this is not true for general Dirac structures. 
Our quantization of Dirac structures will make essential use of the following result of elementary linear algebra.

Lemma 2.6. For any $\Gamma \in \mathcal{D}_{n}$, there is a subset $I$ with $|I|=n-C(\Gamma)$ for which $\mathbb{R}^{I}$ is complementary to $N(\Gamma)$.

We will also use:

Lemma 2.7. If $\mathbb{R}^{I^{\prime}}$ is complementary to $C(\Gamma)$ (so that, in particular, $|I|=N(\Gamma)$ ), then $N\left(\sigma_{I}(\Gamma)\right)=0$; i.e. $\sigma_{I}(\Gamma)$ is a Poisson structure. Conversely, if $|I|=N(\Gamma)$ and $\sigma_{I}(\Gamma)$ is a Poisson structure, then $\mathbb{R}^{I^{\prime}}$ is complementary to $C(\Gamma)$.

Proof. Taking annihilators in the direct sum decomposition

$$
C(\Gamma) \oplus \mathbb{R}^{I^{\prime}}=\mathbb{R}^{n}
$$

and using (5) gives the dual decomposition

$$
p_{*}(\Gamma) \oplus \mathbb{R}^{I^{*}}=\mathbb{R}^{n *}
$$

Now $\sigma_{I}(\Gamma)$ is a Poisson structure if it has zero intersection with $\mathbb{R}^{n}$ or, equivalently, if $\Gamma \cap \sigma_{I}\left(\mathbb{R}^{n}\right)=\{0\}$.

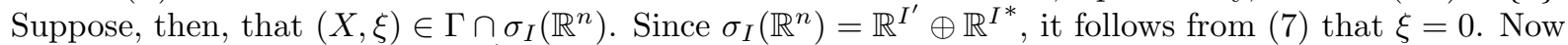
we have $(X, 0) \in \Gamma$ with $X \in \mathbb{R}^{I^{\prime}}$, which by (6) implies that $X=0$ as well.

For the converse, it suffices to prove that, if $\sigma_{I}(\Gamma)$ is a Poisson structure, then $\mathbb{R}^{I^{\prime}} \cap C(\Gamma)=\{0\}$. But if $X$ is an element of this intersection, then $(X, 0) \in \Gamma$, and $\sigma^{I}(X, 0)=(X, 0)$. Since $\sigma_{I}(\Gamma)$ is a Poisson structure, we must have $X=0$.

We will see below that, when the constant Dirac structure on $\mathbb{T}^{n}$ given by $\Gamma$ is quantized by our method, the resulting algebra is just that obtained by quantizing the Poisson structure $\sigma_{I}(\Gamma)$.

In general, there are many $\sigma_{I}$ 's which can convert a given Dirac structure to a Poisson structure. However, the ones with minimal length are those given by Lemma 2.7 This follows from the following lemma and its corollary.

Lemma 2.8. For any Dirac structure $\Gamma$ and any $i, N\left(\sigma_{\{i\}}(\Gamma)\right)=N(\Gamma) \pm 1$.

Proof. It follows from (5) that the statement is true if $\operatorname{dim} p_{*}\left(\sigma_{\{i\}}(\Gamma)\right)=\operatorname{dim} p_{*}(\Gamma) \pm 1$. Writing $\sigma_{\{i\}}$ as $1+R$, where $R$ has rank 2 , we find that

$$
p_{*}\left(\sigma_{\{i\}}(\Gamma)\right)=p_{*}((1+R)(\Gamma)) \subseteq p_{*}(\Gamma)+p_{*} R(\Gamma)
$$

Since $p_{*} R(\Gamma)$ has dimension at most 2 , it follows that $\operatorname{dim} p_{*}\left(\sigma_{\{i\}}(\Gamma)\right) \leq \operatorname{dim} p_{*}(\Gamma)+2$. Since $\sigma_{\{i\}}$ is an involution, we also have $\operatorname{dim} p_{*}\left(\sigma_{\{i\}}(\Gamma)\right) \geq \operatorname{dim} p_{*}(\Gamma)-2$, and the result now follows from Lemma 2.2

Corollary 2.9. If $\sigma_{I}(\Gamma)$ is the a Poisson structure, then $|I| \geq N(\Gamma)$.

\section{Quantization}

In this section, we will define the quantization of a constant Dirac structure on a torus as a Morita equivalence class of algebras. In fact, we will see that quantizations can all be realized as quantizations of constant Poisson structures. ${ }^{2}$

From now on, we will identify each constant Dirac structure on $\mathbb{T}^{n}$ with the corresponding $\Gamma \in \mathcal{D}_{n}$. The subspace $C(\Gamma)$ then determines the direction of the characteristic foliation.

It follows from Lemma 2.6 that $C(\Gamma)$ has a complementary subspace $\widetilde{M}$ having a basis with rational components; we will often work with complements of the special form given by selecting a subset of the coordinate axes, but any rational complement can be put in this form by a change of coordinates in $G L(n, \mathbb{Z})$. The projection of any rational complement into $\mathbb{T}^{n}$ is a compact subtorus $M$ which is a complete transversal to the characteristic foliation.

\footnotetext{
${ }^{2}$ We are indebted to Marc Rieffel for pointing this out.
} 
The Dirac structure $\Gamma$ induces a transverse Poisson structure $\Pi_{\Gamma, M}$ on the transversal torus $M$. We will construct an algebra $\mathcal{A}_{\Gamma, M}$ by using this Poisson structure to "quantize" the groupoid algebra of the restriction to $M$ of the fundamental groupoid along the leaves of the characteristic foliation of $\Gamma$. The full fundamental groupoid is naturally isomorphic to the transformation groupoid associated with the translation action of $C(\Gamma)$ on $\mathbb{T}^{n}$; its restriction to $M$ becomes the transformation groupoid of the translation action of a lattice. The subspace and lattice may be identified with $\mathbb{R}^{n} / \widetilde{M} \cong \mathbb{R}^{k}$ and its integer lattice $\mathbb{Z}^{k}$ respectively. In more geometric terms, we may consider $\mathbb{T}^{n}$ as a principal $M$ bundle over the quotient torus $\mathbb{T}^{n} / M$ whose fundamental group is the lattice $\mathbb{Z}^{k}$. The characteristic foliation is a flat connection on this principal bundle, and the homomorphism $\mathbb{Z}^{k} \rightarrow M$ giving the translation action is the holonomy of this foliation.

The translation action on the torus $M$ clearly induces an action on the quantum torus algebra $\mathcal{A}_{\Pi_{\Gamma, M}}$, which enables us to make the following definition.

Definition 3.1. With notation and terminology as above, we define the algebra $\mathcal{A}_{\Gamma, M}$ to be the crossed product $C^{*}$-algebra $\mathcal{A}_{\Pi_{\Gamma, M}} \times \mathbb{Z}^{k}$.

Remark 3.2. We may think of the crossed product $\mathcal{A}_{\Gamma, M}=\mathcal{A}_{\Pi_{\Gamma, M}} \times \mathbb{Z}^{k}$ as a "quantization" of the algebra $C(M) \times \mathbb{Z}^{k}$. The latter is the groupoid algebra of the transformation groupoid $M \times \mathbb{Z}^{k} \Longrightarrow M$ associated to the translation action of $\mathbb{Z}^{k}$ on $M$. We may therefore think of $\mathcal{A}_{\Gamma, M}$ as the groupoid algebra (but not the function algebra) of a quantum groupoid. It may thus be considered as a (strict) deformation quantization of a noncommutative algebra, in the spirit of [1] and [20. We refer to [18] for more details.

The construction just described becomes much more concrete when the complement $\widetilde{M}$ is taken to be of the form $R^{I^{\prime}}$ for suitably chosen $I^{\prime}$. (As we remarked earlier, any complement can be put in this form by a transformation in $G L(n, \mathbb{Z})$.) $C(\Gamma)$ is then the graph of a linear map $\beta: \mathbb{R}^{I} \rightarrow \mathbb{R}^{I^{\prime}}$ whose composition with the inclusion $\mathbb{Z}^{I} \rightarrow \mathbb{R}^{I}$ and the projection $\mathbb{R}^{I^{\prime}} \rightarrow \mathbb{T}^{I^{\prime}}$ is the holonomy action of $\mathbb{Z}^{I}$ on $\mathbb{T}^{I^{\prime}}$. If, for convenience (and without loss of generality), we number the coordinates so that $I^{\prime}=\{1, \ldots, k\}$, then $\Gamma$ has a basis consisting of the rows of the block matrix.

$$
\left(\begin{array}{llll}
\Pi_{\Gamma, M} & 0 & 1 & -\beta^{t} \\
\beta & 1 & 0 & 0
\end{array}\right) .
$$

where the 1's represent identity matrices.

We may see from this description of $\Gamma$ that the crossed product algebra $\mathcal{A}_{\Gamma, M}$ is generated by $n$ unitary elements which satisfy the commutation relations associated with the Poisson structure

$$
\left(\begin{array}{ll}
\Pi_{\Gamma, M} & -\beta^{t} \\
\beta & 0
\end{array}\right)
$$

The Dirac structure corresponding to this Poisson structure has a basis given by the rows of the block matrix

$$
\left(\begin{array}{llll}
\Pi_{\Gamma, M} & -\beta^{t} & I & 0 \\
\beta & 0 & 0 & I
\end{array}\right)
$$

which is obtained from (8) by interchange of the second and fourth columns, i.e. by the action of the $O(n, n \mid \mathbb{Z})$ element $\sigma_{I}$.

In addition to reproving part of Lemma [2.7] we have thus proven:

Proposition 3.3. If $\Gamma$ is a constant Dirac structure on $\mathbb{T}^{n}$, and if $\mathbb{R}^{I^{\prime}}$ is a complement to $C(\Gamma)$, then the crossed product algebra $\mathcal{A}_{\Gamma, M}$ is isomorphic to the quantum torus algebra $\mathcal{A}_{\sigma_{I}(\Gamma)}$.

Remark 3.4. Although $\mathcal{A}_{\Gamma, M}$ depends only on $\Gamma$ and $M$, the Poisson structure $\sigma_{I}(\Gamma)$ also depends on the choice of a torus complementary to $M$. However, changing the complement changes the Poisson structure by one with integer coefficients, which does not affect the quantization.

Theorem 3.5. The Morita equivalence class of $A_{\Gamma, M}$ is independent of the choice of $M$. 
Proof. We give an outline of the proof, with analytic details to appear in [18. It will be an instance of a quantized version of the fact (see [10]) that Morita equivalent groupoids have Morita equivalent $C^{*}$-algebras.

Let $M_{i}(i=1,2)$ be transversal tori, used in Definition 3.1 to produce algebras $\mathcal{A}_{\Gamma, M_{i}}$. These algebras are quantized versions of the groupoid algebras of the transformation groupoids $G_{i}=M_{i} \times \mathbb{Z}^{k} \Longrightarrow M_{i}$, which are the restrictions to $M_{1}$ and $M_{2}$ of the transformation groupoid $G=\mathbb{T}^{n} \times \mathbb{R}^{k} \Longrightarrow \mathbb{T}^{n}$ associated to the characteristic foliation. An equivalence of groupoids between the $G_{i}$ is given by the "bibundle" $M_{1} \stackrel{\mu_{2}}{\leftarrow} Q \stackrel{\mu_{2}}{\rightarrow} M_{2}$, where $Q$ is the set of morphisms in $G$ with target in $M_{1}$ and source in $M_{2}$, the "moment maps" $\mu_{i}: Q \rightarrow M_{i}$ are given by the target and source maps of $G$, and the (free) actions of $G_{1}$ and $G_{2}$ on $Q$ are given by left and right $G$-multiplication respectively. It is clear that these satisfy the equivalence condition that the orbits of each action are the fibres of the moment map of the other.

If our groupoid algebras were not quantized, the corresponding bimodule would simply be $C(Q)$, as in 10. To adapt to the quantized groupoid algebras, we must also quantize $C(Q)$. We do this by observing first that the $\mu_{i}$ 's are covering maps, so we can pull back the Poisson structure $\Pi_{\Gamma, M_{1}}$ by $\mu_{1}$ to give a Poisson structure $\Pi_{Q}$ on $Q$. Since the Poisson structures on $M_{1}$ and $M_{2}$ are obtained from a invariant transverse bivector to the characteristic foliation, the source map $\mu_{2}$ is then anti-Poisson.

We now use $\Pi_{Q}$ to quantize the functions on $Q$, obtaining an algebra $\mathcal{A}_{Q}$ into which $\mathcal{A}_{\Gamma, M_{1}}$ and the opposite algebra to $\mathcal{A}_{\Gamma, M_{1}}$ embed as mutual commutants, making $\mathcal{A}_{Q}$ into a Morita equivalence bimodule. The Hilbert module structure is a "quantized" version of the one used in [10].

Theorem 3.5 makes the following definition a valid one.

Definition 3.6. If $\Gamma$ is a constant Dirac structure on a torus, the quantization of $\Gamma$ is the Morita equivalence class of algebras containing $\mathcal{A}_{\Gamma, M}$ for any subtorus $M$ which is a complete transversal to the characteristic foliation of $\Gamma$.

Example 3.7. If $\Gamma$ is already a Poisson structure, we must take $M$ to be a point, and so we recover the usual quantization of Poisson structures. On the other hand, if $\Gamma=F \oplus F^{\circ}$, where $F$ is a (constant) foliation, then the transverse Poisson structure is zero, and we obtain the usual foliation algebra associated to a complete transversal. When $n=2$, any odd Dirac structure is of this form, and the quantized algebra is a "rotation algebra." Then $\sigma_{\{1\}}(\Gamma)$ is a Poisson structure whose quantization is the same algebra. This gives a geometric analog of the equivalence between rotation algebras and quantum 2-torus algebras.

Remark 3.8. Although the translation group of $\mathbb{T}^{n}$ preserves the constant Dirac structure $\Gamma$, it does not act on the algebra $\mathcal{A}_{\Gamma, M}$. In some sense which it would be interesting to study, it acts "up to Morita equivalence."

\section{$4 \quad$ Invariance under $O(n, n \mid \mathbb{Z})$ Action}

We are now ready to prove our main theorem. Given the Morita equivalences established in Section 3 the proof will be very short.

Theorem 4.1. If two Dirac structures on $\mathbb{T}^{n}$ are in the same orbit of the $O(n, n \mid \mathbb{Z})$ action, then their quantizations are the same Morita equivalence class.

Proof. In view of Lemma 2.4 it suffices to show that the quantization is unchanged under the action of $\rho(G L(n, \mathbb{Z}))$ and of $\sigma_{\{1\}}$.

Any $A \in G L(n, \mathbb{Z})$ acts on $\mathbb{T}^{n}$, and the invariant nature of our construction implies that the algebras $\mathcal{A}_{\Gamma, M}$ and $\mathcal{A}_{\rho(A)(\Gamma), A(M)}$ are isomorphic.

For $\sigma_{\{1\}}$, our argument is based on the following diagram.

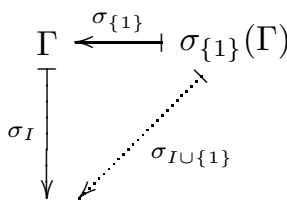

$\sigma_{I}(\Gamma)$ 
To prove that $\Gamma$ and $\sigma_{\{1\}} \Gamma$ have the same quantization, we first apply Lemma 2.8 to conclude that $N(\Gamma)$ and $N\left(\sigma_{\{1\}}(\Gamma)\right)$ differ by \pm 1 , which allows us to assume that $N\left(\sigma_{\{1\}}(\Gamma)\right)=N(\Gamma)+1$.

We now apply Proposition 3.3 and represent the quantization of $\Gamma$ by the quantum torus algebra $\mathcal{A}_{\sigma_{I}(\Gamma)}$, where $|I|=N(\Gamma)$ and $\sigma_{I}(\Gamma)$ is a Poisson structure. Now $\sigma_{I \cup\{1\}}\left(\sigma_{\{1\}}(\Gamma)\right)=\sigma_{I}(\Gamma)$ is a Poisson structure, so by Lemma [2.8 and Corollary [2.9] $|I \cup\{1\}|=N\left(\sigma_{\{1\}}(\Gamma)\right)$, and hence the quantization of $\sigma_{\{1\}}(\Gamma)$ is also represented by $\mathcal{A}_{\sigma_{I}(\Gamma)}$.

In view of the $O(n, n \mid \mathbb{Z})$ equivariance of the identification of Poisson structures with their graphs, we have:

Corollary 4.2. If $\Pi \in \mathcal{T}_{n}$ and $g \in S O(n, n \mid \mathbb{Z})$ are such that $g \cdot \Pi$ is defined, then the algebras $\mathcal{A}_{\Pi}$ and $\mathcal{A}_{g}$. are Morita equivalent.

This is exactly extension of Theorem [1.1] conjectured in [16] and proved in [8].

\section{Final remarks}

Remark 5.1. In [17], A. Schwarz defines a refined notion of Morita equivalence - complete Morita equivalence, which requires that there be a connection on the Hilbert bimodule, compatible with constant curvature connections on the noncommutative tori of both sides. He showed that any two noncommutative tori must sit in the same orbit of $S O(n, n \mid \mathbb{Z})$ if they are completely Morita equivalent. The statement of our main theorem 4.1 is also true for complete Morita equivalence. The key point is that the bimodule constructed in [18 can be identified with a Heisenberg module as defined by Rieffel in 14. The complete Morita equivalence then follows from Rieffel's result on Heisenberg modules . The relation between our bimodule and the bimodule constructed in [16] will be studied in [18].

Remark 5.2. Our proof of Theorem 4.1 is not optimal. We would like to find a uniform construction which takes an $O(n, n \mid \mathbb{Z})$ element and produces a Morita equivalence bimodule. The possibility of such a construction is related to the structure of the "Picard groupoid" in which the objects are pairs $(\Gamma, M)$ and the morphisms are isomorphism classes of Morita equivalence bimodules between the corresponding algebras.

Remark 5.3. Our approach in this paper may be related to open string theory in the following way. In string theory, a subtorus $M$ may be considered as a " $k$-brane." Strings ending on this brane are geodesics perpendicular to $M$ with respect to some background metric. Therefore they lie in a foliation transverse to $M$. If we are also given a " $B$-field" on $M$, represented by a constant bivector, then there is a unique Dirac structure $\Gamma$ on the torus for which the characteristic foliation $C(\Gamma)$ is the given one and the transverse Poisson structure $\Pi_{\Gamma, M}$ is the $B$-field. The field theory of of this open string is described (see, for example, [6]) by the crossed product algebra in Definition 3.1

The action of $\sigma_{\{i\}}$, can be seen as a t-duality in the $i$-th direction. Lemma 2.4 in this paper shows that $O(n, n \mid \mathbb{Z})$ is the full group generated by t-duality. And Theorem 4.1] shows that different open string theories related by t-duality are Morita equivalent.

Remark 5.4. There is another geometrical explanation of our Definition 3.1 which shows a relation to algebraic geometry and homological mirror symmetry.

For a torus $\mathbb{T}^{n}$, we consider the moduli space of flat connections on its trivial line bundle. Each such connection can be identified with its holonomy, a homomorphism from the fundamental group of $\mathbb{T}^{n}$ to the structure group $\mathbb{T}^{1}$. It is easy to see that the set of all flat connections is an $n$-torus, but its typical tangent space is naturally identified with the typical cotangent space of $\mathbb{T}^{n}$. Therefore, we call this new torus the dual torus of $\mathbb{T}^{n}$, written as $\widehat{\mathbb{T}^{n}}$.

When a Dirac structure $\Gamma \in \mathcal{D}_{n}$ has positive nullity, it is not a Poisson structure. However, when we fix a transversal $M$ to $C(\Gamma)$, it is not difficult to see that $\Gamma$ defines a Poisson structure on an n-dimensional subtorus $M \times M^{0}$ in $\mathbb{T}^{n} \times \widehat{\mathbb{T}^{n}}$. In particular, when $\Gamma$ is $\{0\} \times \mathbb{R}^{n}$, it defines the zero Poisson structure on $\widehat{\mathbb{T}^{n}}$.

The group $O(n, n \mid \mathbb{Z})$ acts on $\mathbb{T}^{n} \times \widehat{\mathbb{T}^{n}}$ naturally. The subgroup $\rho(G L(n, \mathbb{Z}))$ fixes the product decomposition of $\mathbb{T}^{n} \times \widehat{\mathbb{T}^{n}}$, while $\sigma_{\{i\}}$ maps the $i$-th component torus to its dual. 
The picture above can be seen as a real analog of the theory of Mukai transformations [11] of abelian varieties. We hope to elucidate this connection and its relation to Kontsevich's homological mirror symmetry [7] in a future publication.

\section{References}

[1] Block, J., and Getzler, E., Quantization of foliations, Proceedings of the XXth International Conference on Differential Geometric Methods in Theoretical Physics, Vol. 1, 2 (New York, 1991), World Scientific, River Edge, NJ, 1992, 471-487.

[2] Connes, A., Noncommutative Geometry, Academic Press, 1994.

[3] Connes, A., Douglas, M.R., and Schwarz, A., Noncommutative Geometry and Matrix Theory: Compactification on Tori, J. High Energy Phys., 9802(1998)003

[4] Courant, T.J., Dirac manifolds, Trans. A.M.S. 319 (1990), 631-661.

[5] Elliott, G. A., On the K-theory of the $C^{*}$ algebras generated by a projective representation of a torsion-free discrete abelian group, Operator Algebras and Group Representations, 157-184, Pitman, London, 1984.

[6] Kajiura, H., Kronecker foliation, D1-branes and Morita equivalence of noncommutative two-tori, $J$. High Energy Phys. 8 (2002), no. 50.

[7] Kontsevich, M., Homological algebra of mirror symmetry. Proceedings of the International Congress of Mathematicians, Vol. 1, 2 (Zürich, 1994) 120-139, Birkhuser, Basel, 1995.

[8] Li, H., Strong Morita equivalence of higher-dimensional noncommutative tori, preprint math.OA/0303123

[9] Lizzi, F., and Szabo, R., Noncommutative Geometry and String Duality, Corfu Summer Institute on Elementary Particle Physics (Kerkyra, 1998, PRHEP-corfu98/073, 17 pp. (electronic), J. High Energy Phys. Conf. Proc., J. High Energy Phys., Trieste, 1999.

[10] Muhly, P.S., Renault, J.N., and Williams, D.P., Equivalence and isomorphism for groupoid $C^{*}-$ algebras, J. Operator Theory 17 (1987), 3-22.

[11] Mukai, S., Duality between $D(X)$ and $D(\hat{X})$ with its application to Picard sheaves, Nagoya Math. J. 81 (1981), 153-175.

[12] Rieffel, M.A., Morita equivalence for $C^{*}$-algebras and $W^{*}$-algebras, J. Pure Appl. Algebra 5 (1974), 51-96.

[13] Rieffel, M.A., $C^{*}$-algebras associated with irrational rotations, Pacific. J. Math. 93 (1981), 415-429.

[14] Rieffel, M.A., Projective modules over higher-dimensional non-commutative noncommutative tori, Canadian J.Math.40(1988), 257-338.

[15] Rieffel, M.A., Deformation quantization of Heisenberg manifolds, Commun. Math. Phys. 122 (1989), 531-562.

[16] Rieffel, M.A., and Schwarz, A., Morita equivalence of multidimensional noncommutative tori, Int. J. Math. 10 (1999), 289-299.

[17] Schwarz, A., Morita equivalence and duality, Lett. Math. Phys. 50 (1999), 309-321.

[18] Tang, X., Quantization of pseudo-étale groupoids (in preparation).

[19] Weinstein, A., Symplectic groupoids, geometric quantization, and irrational rotation algebras, Symplectic geometry, groupoids, and integrable systems, Séminaire sud-Rhodanien de géométrie à Berkeley (1989), P. Dazord and A. Weinstein, eds., Springer-MSRI Series (1991), 281-290.

[20] Xu, P., Noncommutative Poisson algebras, Amer. J. Math. 116 (1994), 101-125. 